\title{
Hypogonadism and Hepatic Steatosis in Heterozygous Hemochromatosis
}

ISSN: 2637-7632

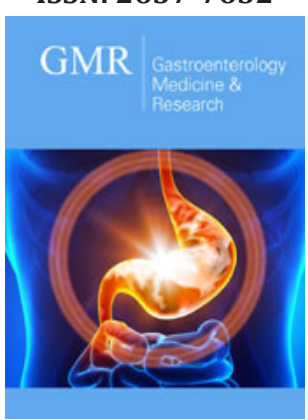

*Corresponding author: Lawrence B Cohen, Associate Professor, Canada

Submission: 眥June 11, 2020

Published: 㘹June 29, 2020

Volume 4 - Issue 5

How to cite this article: Pattni C, Halperin I, Cohen LB. Hypogonadism and Hepatic Steatosis in Heterozygous Hemochromatosis. Gastro Med Res. 4(5). GMR.000596. 2020.

DOI: 10.31031/GMR.2020.04.000596

Copyright@ Cohen LB, This article is distributed under the terms of the Creative Commons Attribution 4.0 International License, which permits unrestricted use and redistribution provided that the original author and source are credited.

\author{
Pattni $\mathrm{C}^{1}$, Halperin $\mathrm{I}^{2}$ and Cohen $\mathrm{LB}^{3 *}$ \\ ${ }^{1}$ Division of Gastroenterology, Canada \\ ${ }^{2}$ Division of Endocrinology, Canada \\ ${ }^{3}$ Associate Professor, Canada
}

\begin{abstract}
A 52-year-old gentleman with a compound heterozygote genotype (C282Y/H63D) of hereditary hemochromatosis $(\mathrm{HH})$ complicated by hypogonadism and hepatic steatosis underwent successful resolution of these clinical problems with phlebotomy and hormone replacement therapy. The patient had no features of metabolic syndrome nor did he consume significant amounts of alcohol. Investigations revealed normal $\mathrm{HbA1c}$, lipid panel, and liver biochemistry. Comprehensive review of current literature indicates that the clinical significance of compound heterozygosity for $\mathrm{HH}$ is controversial and that other aetiologies for hepatic steatosis and hypogonadism must be ruled out initially. Low testosterone levels have been associated with hepatic steatosis in non-alcoholic fatty liver disease (NAFLD) attributed to low circulating sex hormone binding globulin. There is also an association between HH compound heterozygosity and the development of NAFLD and hepatocellular carcinoma (HCC) with or without cirrhosis. Depletion of iron stores by phlebotomy and complete abstinence from alcohol likely contributed to resolution this patient's severe hepatic steatosis.
\end{abstract}

\section{Introduction}

Hereditary hemochromatosis $(\mathrm{HH})$ is one of the most common genetic disorders among those of northern European descent [1]. With recent advances in the wide availability of molecular diagnostics for genotyping of this disorder, there has been a need for understanding the clinical relevance of the various $\mathrm{HH}$ mutations. We report a case of a man who was found to be an HFE gene compound heterozygote (C282Y/H63D) after presenting with fatigue and loss of libido in the context of low testosterone levels, mild serum ferritin (SF) elevation, and hepatic steatosis. The possible implications of this HFE gene mutation and ethology of clinical manifestations are presented with a brief review of the available literature.

\section{Objectives}

1. Clinical relevance of compound heterozygous HH genotype.

2. Relationship between HH, hypogonadism, and NAFLD.

3. Management considerations for patients with HH and NAFLD.

3. Case Report

A healthy 52-year-old man was referred to a Gastroenterology clinic by his endocrinologist to explore the significance of hepatic steatosis associated with findings of low testosterone levels and heterozygous hemochromatosis genotype. The patient initially presented to his family physician for evaluation of fatigue and diminished libido, associated with a low testosterone level, which had resolved with subsequent supplementation. There was no additional medical or surgical history and the patient had no manifestations of metabolic syndrome or other endocrinopathies. Alcohol consumption consisted of 3-5 glasses of wine weekly. On family history, the patient's father may have had a state of iron overload, though 
further documentation was not available. On physical examination, he had a BMI of $26.5 \mathrm{~kg} / \mathrm{m} 2$ with waist circumference was $96 \mathrm{~cm}$, below the $102 \mathrm{~cm}$ threshold for obesity in men. There were no peripheral stigmata of chronic liver disease or acanthosis nigricans. All other systems were otherwise normal.

Review of laboratory investigations showed a normal complete blood count (CBC), SF 546ug/l (upper limit of normal $400 \mathrm{ug} / \mathrm{l}$ ), and iron saturation 0.45 . Liver biochemistry (AST, ALT \&ALP) and markers of synthetic function were all within normal limits. Hemochromatosis genotyping yielded C282Y/H63D heterozygosity. Hypogonadism workup provided by the patient's endocrinologist demonstrated low testosterone levels, low LH, lownormal FSH, and low basal cortisol levels. All other pituitary and hypothalamic hormones were within normal limits including TSH and prolactin. ACTH stimulation test was conducted and ruled out a central adrenal insufficiency. With testosterone supplementation, hormone levels remained stable over 1 year of follow-up with his endocrinologist. Initial MRI Brain showed a 7X8mm abnormality thought to be a pituitary microadenoma which was not substantiated on subsequent imaging studies. An initial Fibro scan, in lieu of a liver biopsy, was arranged demonstrating Grade 3/3 hepatic steatosis (NAFLD Activity Score), and Stage 1/4 fibrosis (Kleiner classification) [2].

As per the 2019ACG guidelines for hereditary hemochromatosis, we recommended monitoring $\mathrm{CBC}$, liver, and iron profiles with monthly phlebotomy to keep serum ferritin in the low-normal range without compromising his haemoglobin. At the end of treatment follow up, the ferritin was reduced to $146 \mathrm{ug} / \mathrm{l}$ and iron saturation was reduced modestly to 0.34 . Reduction or elimination of alcohol consumption was advised, along with restriction of Tylenol to $2 \mathrm{~g} /$ day if needed. The patient was followed up on a yearly basis by his endocrinologist and family doctor and has been compliant with treatment recommendations. He is currently well with stable hypothalamic/pituitary hormone levels and 1-year follow-up Fibro scan showing diminished steatosis (Grade 2/3) with no evidence for fibrosis when compared to the baseline study [2].

\section{Discussion}

$\mathrm{HH}$ is an iron overload disorder characterized by excessive iron absorption, due to deficiency of hepcidin [3]. HFE, the hemochromatosis gene, is located on chromosome 6 and encodes the HFE protein, of which the most common mutation is an amino acid substitution known as C282Y followed by H63D [4]. A compound heterozygous hemochromatosis genotype pattern (C282Y/H63D) carries a low risk for developing overt $\mathrm{HH}$ and related adverse sequelae $[4,5]$. They tend to have evidence of hepatic steatosis or chronic hepatitis with lower total body iron stores compared to C282Y homozygotes, though typically in the presence of comorbid factors such as hepatic steatosis, obesity, and significant alcohol intake [6,7]. Furthermore, a recent systematic review and metaanalysis demonstrated a positive association between compound heterozygosity for C282Y/H63D and the risk of NAFLD and HCC, without liver cirrhosis [8] in contrast to prior studies $[9,10]$. The 2019 ACG guidelines for $\mathrm{HH}$ also don't reflect these findings [8] as their official recommendation is not to screen for HCC in patients with $\mathrm{HH}$ and Stage 3 or less fibrosis [1].

Hypogonadism has been shown to be the most common nondiabetic manifestation of homozygous hemochromatosis but has not been implicated in the heterozygous variant to a significant degree [11]. However, HFE and transferrin gene polymorphisms have been shown to effect the hypothalamic-pituitary- gonadal (HPG) axis in a case-control association study of 127 infertile men [12]. Conversely, low testosterone levels have been associated with hepatic steatosis in NAFLD independent of the presence of diabetes mellitus, obesity, or hyperlipidaemia presumably via low circulating sex hormone binding globulin $[13,14]$. Furthermore, higher testosterone and sex hormone binding globulin levels in men are associated with a reduced risk of hepatic steatosis and elevated AST/ALT ratios, independent of age, smoking, alcohol consumption and physical activity [15]. Therefore, in our patient, hypogonadism can be independently associated with $\mathrm{HH}$, though less likely with compound heterozygosity and lower SF levels, or with NAFLD/ hepatic steatosis independent of the presence of the metabolic syndrome phenotype. Investigating sex hormone binding globulin levels may be a way to distinguish between the two possible aetiologies. Only one similar case report was found published by a Dutch group in 2000 describing a 24-year-old man presenting with loss of libido and erectile dysfunction as the initial symptoms of hemochromatosis, though with a non-classical genotype [16]. In our patient, the SF level was very mildly elevated which is not commonly associated with iron-overload related disease manifestations. Given the overview of the literature, the clinical relevance of a C282Y/ H63D mutation is controversial. Additionally, our patient does not demonstrate the classic metabolic syndrome phenotype given the lack of insulin resistance, obesity, and abdominal adiposity. In the context of his HFE mutation and lack of comorbidities, there is a lower risk of development of hepatic fibrosis and cirrhosis from his current state of steatosis as further demonstrated by his follow-up Fibro Scan. However, given the positive association of his mutation with NAFLD and HCC without liver cirrhosis/fibrosis along with the lack of clarity regarding this in ACG's 2019 guideline, ongoing screening for HCC will need to be an educated discussion between the provider and the patient.

\section{Role of the Sponsor}

No funding organization had any role in the design and conduct of the study, collection, management, analysis and interpretation of the data; and preparation, review, or approval of the manuscript.

\section{Consent}

Informed consent was obtained from the patient prior to submission of case report. Signed consent form can be provided to journal if necessary.

\section{References}

1. Kowdley KV, Brown KE, Ahn J, Sundaram V (2019) ACG clinical guideline: Hereditary hemochromatosis. Am J Gastroenterol 114(8): 1202-1218.

2. Kwok R, Tse YK, Wong GLH, Ha Y, Lee AU, et al. (2014) Systematic review with meta-analysis: Non-invasive assessment of non-alcoholic fatty liver 
disease--the role of transient elastography and plasma cytokeratin-18 fragments. Aliment Pharmacol Ther 39(3): 254-269.

3. Brissot P, Pietrangelo A, Adams PC, McLaren CE, Loreal O, et al. (2018) Haemochromatosis. Nat Rev Dis Prim 4: 18016.

4. Moirand R, Jouanolle AM, Brissot P, Le Gall JY, David V, et al. (1999) Phenotypic expression of HFE mutations: A French study of 1110 unrelated iron-overloaded patients and relatives. Gastroenterology 116(2): 372377.

5. Powell LW, Seckington RC, Deugnier Y (2016) Haemochromatosis. Lancet 388(10045): 706-716.

6. Walsh A, Dixon JL, Ramm GA, Hewett DG, Lincoln DJ, et al. (2006) The clinical relevance of compound heterozygosity for the c282y and h63d substitutions in hemochromatosis. Clin Gastroenterol Hepatol 4(11): 1403-1410.

7. Cheng R, Barton JC, Morrison ED, Phatak PD, Krawitt ED, et al. (2009) Differences in hepatic phenotype between hemochromatosis patients with HFE C282Y homozygosity and other HFE genotypes. J Clin Gastroenterol 43(6): 569-573.

8. Ye Q Qian BX, Yin WL, Wang FM, Han T (2016) Association between the HFE C282Y, H63D polymorphisms and the risks of non-alcoholic fatty liver disease, liver cirrhosis and hepatocellular carcinoma: An updated systematic review and meta-analysis of 5,758 cases and 14,741 controls. PLoS One 11(9): e0163423.

9. Gallego CJ, Burt A, Sundaresan AS, Ye ZI, Shaw C, et al. (2005) Penetrance of hemochromatosis in HFE genotypes resulting in p. Cys282Tyr and p. [Cys282Tyr]; [His63Asp] in the emerge Network. Am J Hum Genet 97(4): 512-520.
10. Cauza E, Peck-Radosavljevic M, Ulrich-Pur H, Datz C, Gschwantler M, et al. (2003) Mutations of the HFE gene in patients with hepatocellular carcinoma. Am J Gastroenterol 98(2): 442-447.

11. McDermott JH, Walsh CH (2005) Hypogonadism in hereditary hemochromatosis. J Clin Endocrinol Metab 90(4): 2451-2455.

12. Tomljanovic AB, Vlastelic I, Radojcic Badovinac A, Cizmarevic NS, Nadalin S, et al. (2009) The impact of hemochromatosis mutations and transferrin genotype on gonadotropin serum levels in infertile men. Fertil Steril 91(5): 1793-1800.

13. Yim JY, Kim J, Kim D, Ahmed A (2018) Serum testosterone and non-alcoholic fatty liver disease in men and women in the US. Liver Int 38(11): 2051-2059.

14. Jaruvongvanich V, Sanguankeo A, Riangwiwat T, Upala S (2017) Testosterone, sex hormone-binding globulin and nonalcoholic fatty liver disease: A systematic review and meta-analysis. Ann Hepatol 16(3): 382394.

15. Yang Q, Wu K, Zhuang Y, et al. (2018) Association of total testosterone, free testosterone, bioavailable testosterone and sex hormone-binding globulin with hepatic steatosis and the ratio of aspartate aminotransferase to alanine aminotransferase. Endocr J 65(9): 915-921.

16. Berent R, Allinger S, Höbling W, Auer J, Knoflach P (2000) A 24-year-old patient with decreased libido and erectile dysfunction as initial manifestations of hemochromatosis. Dtsch Med Wochenschr 125(48): 14661468.

For possible submissions Click below:

Submit Article 